\title{
Colourful work on quarks scoops triple crown
}

Philip Ball

While the Nobel prize for medicine has been awarded for work on smell, this year's physics Nobel stems from research on colour.

But it is not exactly colour as we know it. The prize has been scooped for work on 'colour interaction': the poetic way in which physicists refer to the strong nuclear force, which binds together the fundamental particles called quarks that make up protons and neutrons in an atomic nucleus.

The prize is shared by David Gross of the University of California, Santa Barbara, David Politzer of the California Institute of Technology and Frank Wilczek of the Massachusetts Institute of Technology for their work illuminating the bizarre nature of this force - and making it calculable, at least some of the time.

"It's fantastic news," says particle physicist Jeff Forshaw of the University of Manchester, UK. "Until they came along, we were really struggling to understand the strong force."

Of the four basic forces in nature - gravity, electromagnetism, and the weak and strong nuclear forces - the strong force is particularly counter-intuitive, with the highly unusual property of getting stronger as the quarks get further apart.

This feature, comparable to the behaviour of a rubber band as it is stretched, explains why quarks never appear on their own, but always in combinations. Protons and neutrons each contain a triplet of quarks.

In 1973, Gross, with his graduate student Wilczek, and Politzer published two papers back to back in which they outlined the way

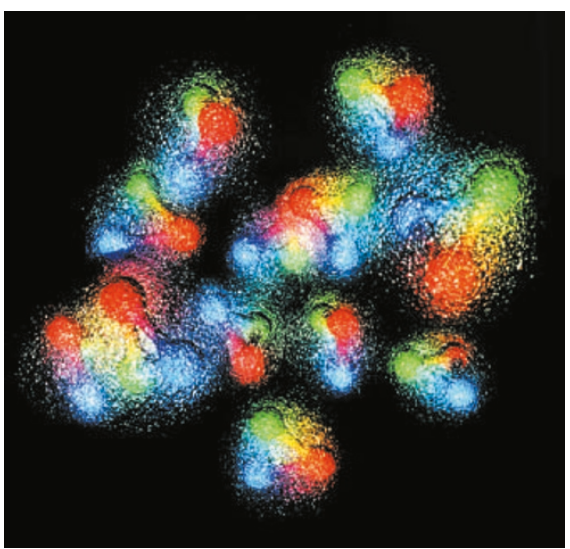

Strong theory: Nobel winners cast light on the gluons that hold nuclear particles together.

the strong force operated (D. J. Gross and F. Wilczek Phys. Rev. Lett. 30, 1343; 1973 and H. D. Politzer Phys. Rev. Lett. 30, 1346; 1973). It had been worked out by then that quarks have a quantized property called 'colour charge', which is analogous to electrical charge but comes in three 'colours': red, blue and green. The theory that attempted to explain the colour interaction was called quantum chromodynamics (QCD).

Before these two papers, no one knew how to solve the equations of QCD theory. The problem was the sheer complexity of the interaction. Just as electrical forces between particles are mediated by photons passing between them, so the strong force is mediated by particles called gluons. But whereas photons have no electrical charge, gluons do have a colour charge, so they interact with each other as well as with quarks. This meant that "the theory was so strongly interacting that it was incalculable", says Forshaw.

Gross, Wilczek and Politzer got round this problem by calculating that the strength of the colour force gets weaker as the particles' energies increase - or equivalently, as the particles get closer together. At sufficiently high energy, the quarks act as though they are 'free'. This became known as 'asymptotic freedom'.

Their work showed particle physicists that if they wanted to understand the strong force they needed to probe it at high energies, where the weakening of the interaction made QCD a testable theory. Studies of highenergy collisions between particles, in particular at the DESY accelerator in Hamburg and the Large Electron-Positron collider at CERN in Geneva, have now verified in great detail the predictions of asymptotic freedom.

Not all aspects of the strong interaction are understood, however. For instance, there is still no way to use QCD to explain some basic properties of quarks at the relatively modest energies characteristic of everyday matter.

But it is precisely the understandable, high-energy region of QCD that researchers are looking at to find a 'theory of everything'. Modifications of QCD at high energies, for example, should be able to describe the Higgs boson, the particle thought to be responsible for giving matter mass.

The Nobel Prize in Chemistry was announced after Nature went to press. For coverage see www.nature.com/news

\section{Harvard ceremony graced by hula-hooping laureates}

\section{Steve Nadis, Boston}

This year's fourteenth Ig Nobel Prize ceremony clashed with the first debate of the US presidential election campaign. But there was no evidence of regret inside the packed auditorium at Harvard University, Massachusetts. For these fans of science that "makes you laugh, and then makes you think", it was George W. Bush and John Kerry who were missing the show.

Presided over by the King and Queen of Swedish Meatballs, as usual, the 30 September event featured Nobel laureates Dudley Herschbach, William Lipscomb and Richard Roberts blowing bullhorns, hulahooping and singing the classic You're Just Too Good To Be True with a karaoke machine.

Herschbach was the delectable prize on offer in the evening's contest to win a date with a Nobel laureate, and Roberts was called on to describe the concept of heredity in just seven words: "Heredity means blame your parents, not yourself," he said.

The Ig Nobel Prize in Medicine went to Steven Stack of Wayne State University in Detroit, Michigan, and James Gundlach of Auburn University, Alabama, for their paper, "The effect of country music on suicide" (Social Forces 71, 211-218; 1992). The physics prize was awarded to Ramesh Balasubramaniam of the University of Ottawa in Ontario, Canada, and Michael Turvey of the University of Connecticut, Storrs, for their sterling work on the dynamics of hula-hooping (Biol. Cybern. 90, 176-190; 2004) - it seems that to keep the plastic ring spinning around the waist, it is vital to move up and down at the knees.

The coveted public-health prize went to Jillian Clarke, a Chicago high-school graduate, for experiments that validated the 'five-second rule', which states that it is safe to eat food dropped on the floor if its stay there was sufficiently brief. And the peace prize went to Daisuke Inoue of Hyogo, Japan, the inventor of karaoke. "Let's party!" he urged the crowd.

Daniel Simons of the University of Illinois at Urbana-Champaign and Christopher Chabris of Harvard University brought home the psychology prize for their report, "Gorillas in our midst", which showed that when observers concentrate on one thing - in their study, people passing a basketball back and forth - they can completely overlook something else - such as a man appearing in a gorilla suit.

And the biology prize went to a collaboration from Canada, Denmark, Scotland and Sweden for demonstrating the role of flatulence in herring communications. Accepting his award, Lawrence Dill of Simon Fraser University, Canada, summed up the evening: "It's been a gas," he said.

www.improb.com/ig 